\title{
Identification of Hemorrhages in Iris Using Hybrid Morphological Method
}

\author{
Robbi Rahim \\ Sekolah Tinggi Ilmu Manajemen Sukma, Kota Medan, Indonesia. \\ robbirahim@ieee.org
}

\begin{abstract}
In the field of ophthalmology, hemorrhage is the term used more often because of increasing diabetic patients. It's a challenge amidst the ophthalmologist to distinguish the hemorrhage from the blood vessels, these lands in various problems. In the past various techniques were employed for the detection of the hemorrhage but they were not so accurate and often encountered misclassification between hemorrhage and blood vessels. Precise detection and classification of hemorrhage and blood vessel is very important in the diagnosis of many problems. This paper depicts a mechanized procedure for recognizing hemorrhages in fundus pictures. The acknowledgment of hemorrhages is one of the critical factors in the early finish of diabetic retinopathy. The algorithm proceeds through several steps such as image enhancement, image subtraction, morphological operations such as image thresholding, image strengthening, image thinning, erosion, morphological closing, image complement to suppress blood vessels and to highlight the hemorrhages..
\end{abstract}

Keywords-Hemorrhage ,Morphological operations, Iris

\section{Introduction}

Retinal hemorrhages are the sporadic leaking of the veins in the retina, which is the film in the back of the eye which is a disarray of the eye where depleting occurs into the retina. The retinal release is achieved by hyper pressure, retinal vein hindrance a blockage of a retinal vein, or diabetes mellitus which makes minimal sensitive veins structure, which are viably hurt. Proliferative retinopathy happens when new veins begin to outline in hurt regions of the retina and may provoke spots, floaters or sudden loss of vision. Non proliferative retinopathy happens when the hurt or spilling veins don't spread [1][2].

At present from the iris infection region, two sorts of pictures are gotten; they are high spatial objectives panchromatic pictures and low spatial objectives multispectral pictures. With the remote distinguishing satellites it is an inconvenient task to get high spatial objectives pictures considering distinctive specific imprisonments of the sensors used in the satellites to get the photos. Picture mix expect a key activity in improving the spatial objectives of the multispectral pictures. Mix is joining significant information from at any rate two pictures into a lone picture [3]. The ensuing picture will be more instructive than any of the data pictures. In the past various blend estimations were proposed and they are called as dish sharpening in light of the fact that the multispectral picture is sharpened by injecting the nuances from the panchromatic picture. In the image mix section substitution system is the convincing method in sharpening multispectral picture. In the part substitution system a segment of the computations are suitable only for explicit sensors yet the monetarily available programming contraptions shows that this methodology is sensible for all open optical panchromatic pictures and multispectral pictures [4].

\section{EXISTING TECHNIQUES}

Micro aneurysm release is the most progressive bizarre injury achieved by diabetic retinopathy and different methodologies have been proposed before [5]. Another methodology for preprocessing and sham positive end which consolidates magnificence of the fundus picture was changed by the non direct curve with brightness estimations of the tone drenching regard (HSV) space then gamma modification is done and channel candidates were perceived using thickness assessment [6]. In this strategy the optic plate is trailed by vein association and high circle power properties in a cost work here geometrical associations of different features of wounds used close by morphological undertakings [7]. This method removed a ton of features from picture areas and picked the subset which best isolated between the red wounds and the retinal background. A multilayer preceptor classifier used to secure last division of red wounds [8]. Here the preprocessing of picture is done then normalization process with histogram detail followed by picture division with optic circle and fovea acknowledgment is done which follows the varieties from the standard [9].

Another flexible CS based picture blend method is suggested that beats the impediments of general CS mix system. This 
procedure uses midway replacement to clear the ghost mutilation and to ensure the main spatial credits notwithstanding to the satellite sensor used. Thusly the spatial nuances can be isolated from the panchromatic picture with no spatial and powerful distortion in each multispectral band. Here our strategy is secluded into two areas. The underlying fragment is advancement of high/low objectives portion picture by fragmentary replacement among panchromatic and multispectral picture. The resulting part is to assemble an adaptable CS mix model to restrain the loathsome dissimilarities among panchromatic and multispectral picture while the spatial objectives is kept up. First the spatially degraded image is obtained from the following equation and which obtained when it is decimated by bi-cubic interpolation [10].

$$
\text { PANS }=\alpha_{\mathrm{O}}+\sum \mathrm{N}_{\alpha \mathrm{n}} \mathrm{M}_{\mathrm{SS}}
$$

Where $\alpha$ is the regression coefficient, $\mathrm{N}$ is the number of spectral bands and MSS nth multispectral image which is bicubically re-sampled with the equivalent size of panchromatic data. Then the initial intensity image is produced using the following equation.

$\mathrm{I}^{\mathrm{S}}=\alpha_{\mathrm{O}}+\sum \mathrm{N}_{\alpha \mathrm{n}} \mathrm{M}_{\mathrm{SS}}$

Where $\mathrm{I}^{\mathrm{S}}$ is the starting power of the picture and $\alpha$ is the relapse coefficient. The relationship coefficient between the low-spatial-goals engineered segment picture and every MS band histogram coordinated with Il is assessed to create the high-goals part picture. Here another high spatial goals segment picture is created utilizing the low spatial goals manufactured segment and the multispectral pictures by altering incomplete substitution [4]. In this way by utilizing every relationship coefficient another high goals part picture is processed utilizing the accompanying condition. Where $\mathrm{CC}_{\mathrm{n}}$ is the relationship coefficient between the low spatial goals part picture and the nth MS band picture and $\mathrm{I}_{\mathrm{h}}$ is the high spatial goals segment picture comparing to the nth MS band picture. What's more, MSS is the nth band histogram coordinated with panchromatic picture. The two stages can be spoken to by the accompanying square charts.

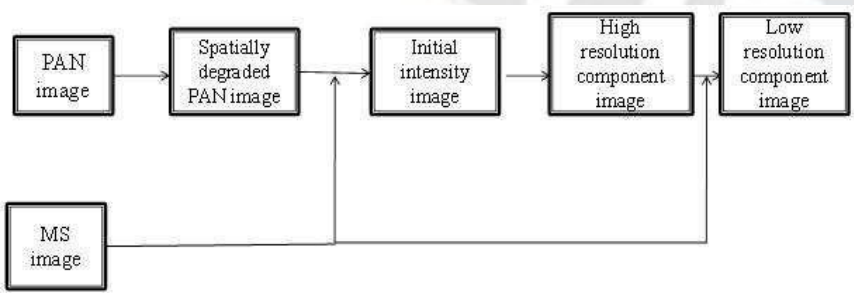

Fig 1: Construction of high and low resolution image component image

The dynamic scope of definite data for each picture diminishes with the decline in the proportion of the standard deviation of the relating band to the mean standard deviation everything being equal. Along these lines the point by point data to be infused into the multispectral picture without loss of ghostly and spatial data. Here the standard deviation proportion has its impact on the ghastly contortion because of the distinctions in standard deviation among the groups. The parameter $\mathrm{p}$ changes and standardizes the high frequencies with the goal that they lie in the comparing dynamic range. The connection coefficient of the above condition modifies the overall size of the high recurrence data to limit the worldwide difference between the high spatial goals picture and each multispectral band. the parameter wn regulates the distinction of dynamic range and worldwide disparity in the infusion of high recurrence. The neighborhood insecurity between the high goals picture and the multispectral band decays the spatial and otherworldly nature of the combined picture. From the accompanying condition the nearby flimsiness modification parameter can be produced.

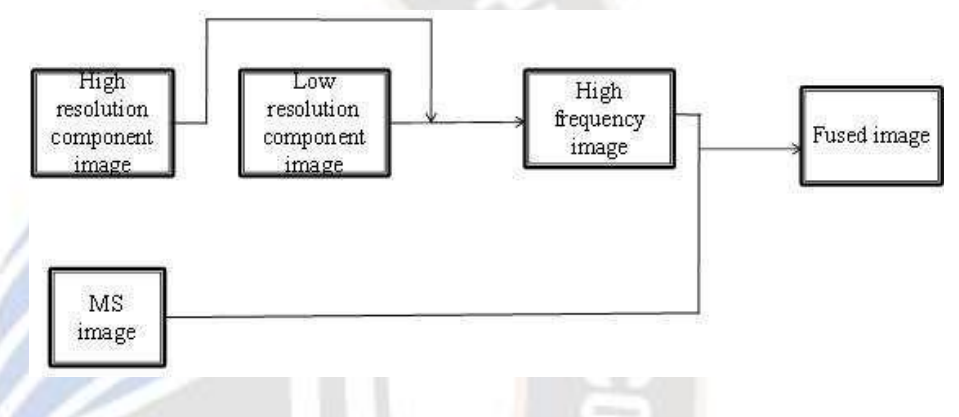

Fig 2: Assembling adaptive CS fusion model

\section{Proposed Methodology}

The scientific morphology comprises the establishment of morphological picture preparing which comprises of set of administrators that change pictures as per portrayals, for example, size, shape, convexity, availability and geodesic separation on both ceaseless and discrete spaces. The input image is shown below.

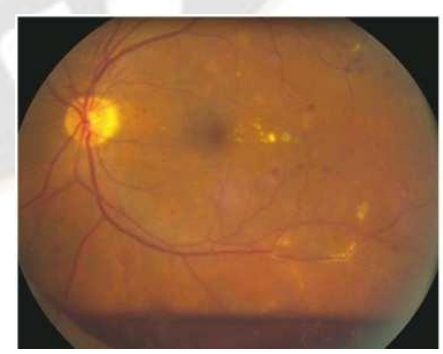

Fig 3: input image of CS fusion model

In photography and processing a grayscale advanced picture is a picture where the estimation of every pixel in a solitary example it conveys just computerized data. Pictures of this sort otherwise called highly contrasting are made only out of shades of dark, fluctuating from dark at the most vulnerable power to white at the most grounded. Grayscale pictures are 
unmistakable from the slightest bit highly contrasting pictures, which with regards to PC imaging are pictures with just two hues high contrast. Dark scale pictures are likewise called monochromatic, meaning the nonattendance of any chromatic variety. Dim scale pictures are regularly the consequence of estimating the force of light at every pixel in a solitary band of the electromagnetic range. The flowchart of the proposed method is shown in figure 4 .

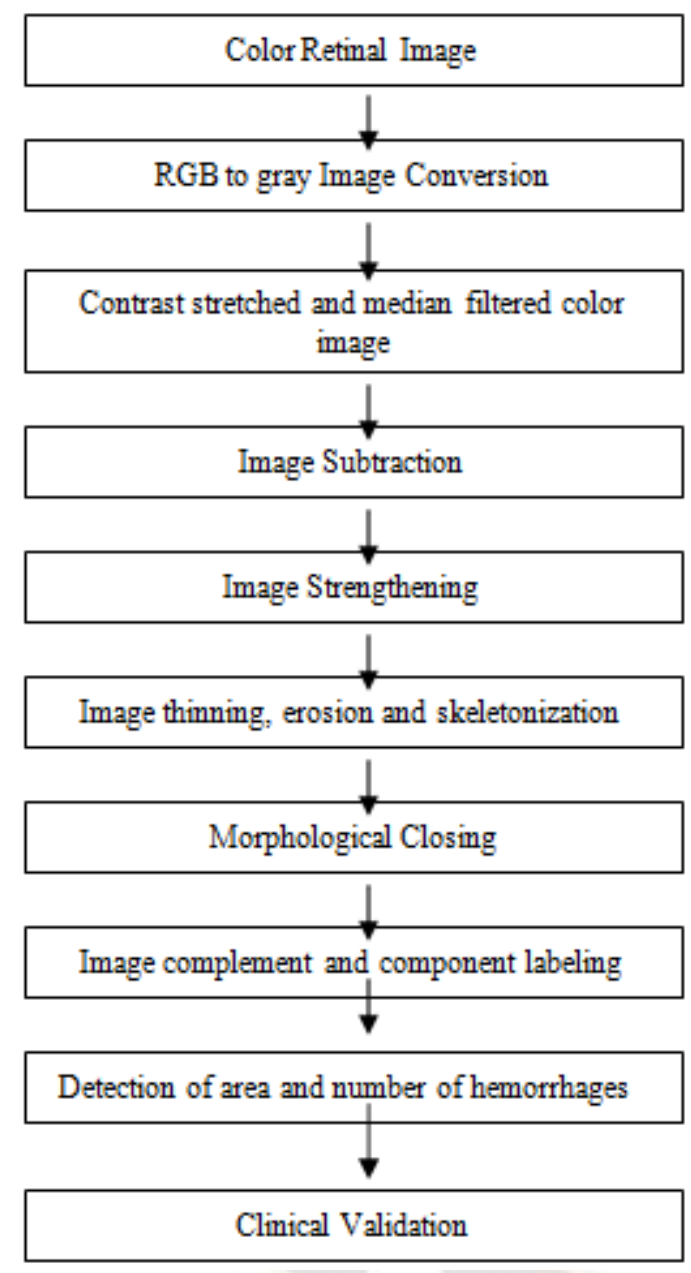

Fig 4: Steps of Hemorrhage Detection

Image preparing, standardization is a procedure that changes the scope of pixel power esteems. Applications incorporate photos with poor difference because of glare; for instance standardization is now and then called differentiate extending. In computerized picture handling it is alluded to as unique range extension. The reason for dynamic range development is generally to bring the picture or other kind of sign into a range that is progressively natural consequently term standardization.

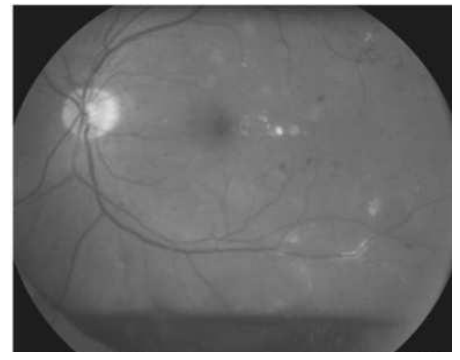

Fig 5: RGB to Gray Scale Conversion

A middle channel is more viable than convolution when the objective is to at the same time lessen commotion and save edges. Middle channel of size more than twice as the vein width is utilized and dim picture is deducted from middle separated picture. With this all structures of retina get offset aside from veins and hemorrhages. This is shown in figure 6.

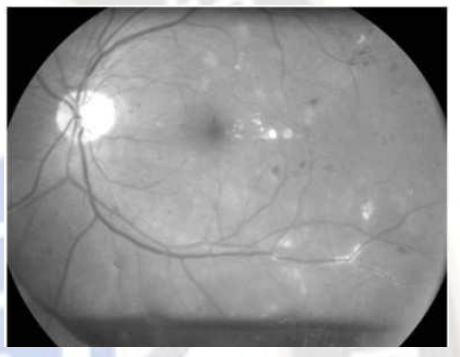

Fig 6: Contrast Stretching

The dim picture is deducted from middle sifted picture so the all structures of retina gets offset with the exception of veins and haemorrhages. The nature of the combined picture can be evaluated by quantitative appraisal. In the quantitative evaluation the combined picture is contrasted and the perfect picture. Here the combined picture ought to be contrasted and the perfect high spatial goals multispectral picture saw by multispectral sensor which creates the high spatial goals picture equivalent to the panchromatic picture. Also, here perfect high spatial goals multispectral picture couldn't got so that spatially debased panchromatic picture and multispectral picture from the first informational indexes are utilized to assess the outcome amount. This quantitative appraisal is not all that exact thus that a novel strategy is known as Quantitative with No Reference (QNR) is utilized to evaluate the combination quality without alluding to a high spatial multispectral picture. The QNR depends on the quality list Q to gauge the neighborhood relationship, luminance, and difference between two pictures. Here it is expected that the inter-band otherworldly quality of the melded information seen as the likeness connection between groups is unaltered after combination.

\section{Simulation Results}

Thresholding is the simplest method of picture division. From a grayscale picture, thresholding can be used to make twofold pictures. During the thresholding system, particular 
pixels in an image are separate as article pixels if their value is more imperative than some edge regard and as establishment pixels. This show is known as cut-off. Varieties fuse limit underneath, which is reverse of edge above edge inside, where a pixel is checked article if its value is between two edges. Article pixel is given an estimation of "1" while an establishment pixel is given an estimation of 0 .

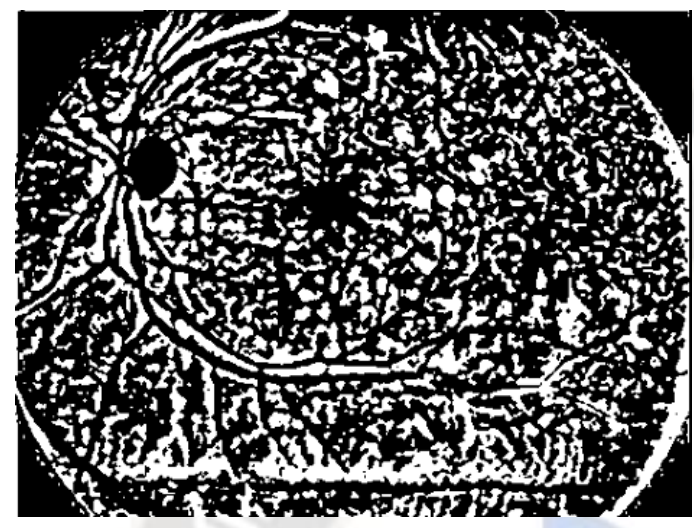

Fig 7: Image after Thersholding operation

Disintegration is one of the two basic activities in morphological picture handling from which all other morphological tasks based. It was initially characterized for paired pictures. In twofold morphology a picture is seen as a subset of a Euclidean space $\mathrm{Rd}$ or the number matrix $\mathrm{Z}_{\mathrm{d}}$ for some measurement $d$. The fundamental thought in twofold morphology is to test a picture with a basic, pre-characterized shape, making inferences on how this shape fits or misses the shapes in the picture. This straightforward 'test' is called organizing component, and is known as a twofold picture. Let us consider a picture An and organizing component is B its disintegration.

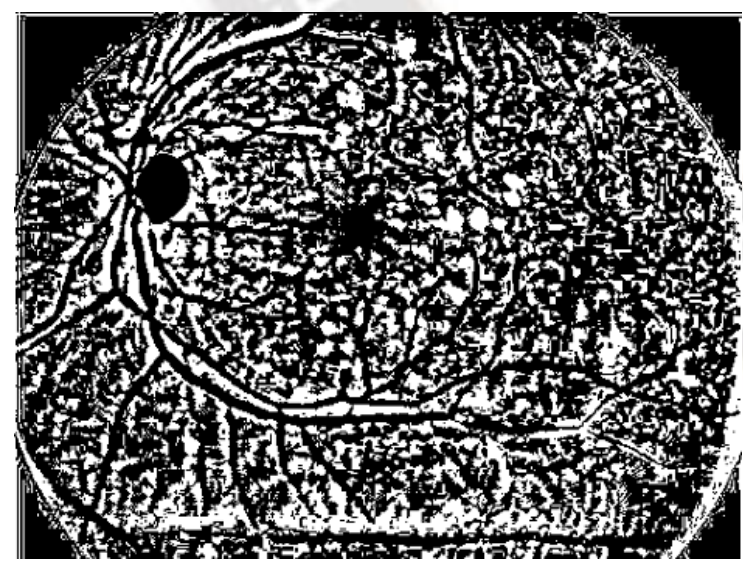

Fig 8: Complemented Image

In double morphology, expansion is a move variation administrator unequivocally identified with minokowski expansion. A twofold picture is seen in scientific morphology as a subset of an Euclidean space Rd or the whole number framework. The picture supplement square processes the supplement of a twofold, power or RGB pictures. For double pictures, the square replaces pixel esteems equivalent to 0 with 1 and pixel esteems equivalent to 1 with 0 . For a force or RGB picture, the square deducts every pixel esteem from the greatest worth that can be spoken to by the information type and yields the distinction. Associated part naming is an algorithmic use of chart hypothesis, where subsets of associated segments are remarkably marked dependent on a given heuristic. Associated segment marking is utilized in PC vision to distinguish associated locales in double advanced pictures, despite the fact that shading pictures and information with higher dimensionality can likewise be prepared.

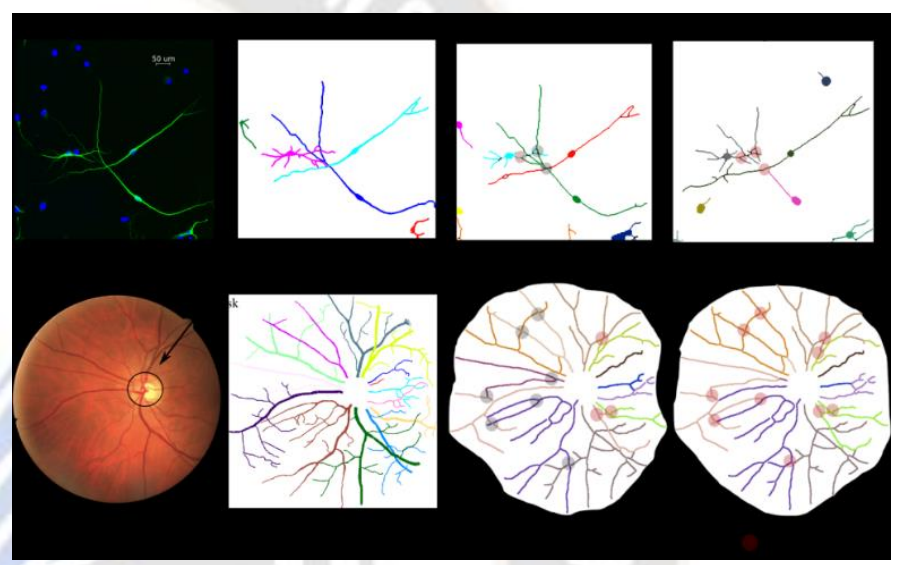

Fig 9: Proposed Hemorrhages in Iris Using hybrid Morphological method

The primary part is the relationship coefficient and its dynamic range is $[-1,-1]$. The subsequent term gauges the comparability that is the mean luminance among $\mathrm{x}$ and $\mathrm{y}$ with a scope of [0-1]. The third term implies how close the complexity among the pictures is and its range is additionally [0-1]. With the goal that the scope of the $Q$ list is [-1-1] and the best estimation of $\mathrm{Q}=1$ is accomplished if $\mathrm{x}=\mathrm{y}$ for all pixels [39]. At the point when the estimation of $Q$ record is more like 1 the more the blended picture is like the first picture. The $\mathrm{Q}$ record is determined utilizing sliding window of size $\mathrm{N}^{*} \mathrm{~N}$ that moves all the lines and sections of the picture so as to build the separation capacity and measure the nearby bending of a combined picture lastly the $\mathrm{Q}$ file is arrived at the midpoint of over all the $\mathrm{Q}$ to ascertain the worldwide score among pictures. In view of the $\mathrm{Q}$ record two contortion lists of the combined picture can be inferred without a reference picture. The main record phantom twisting DB determined from the accompanying condition. 


\section{Conclusion}

In this paper, the iris haemorrhages are recognized using morphological chairmen. The data retinal picture is changed over into dull scale picture. The diminish scale picture is improved using contrast overhaul .The center filtering is applied to redesigned picture to decrease 'salt and pepper racket'. The center channel of size more than twofold the vein width is used and diminish picture is deducted from the center filtered picture .Blood vessels are clearly observable by techniques for picture finding. The particular edge regard is picked with the objective that the veins and hemorrhages are clearly watched. Picture fortifying is done by setting pixel to one if in any event five pixels in its 3 by 3 neighborhood are 1 's regardless set pixels to 0 . Picture lessening, breaking down and skeletonisation is done with the help of sorting out part so the veins are reduced. Then morphological closing which incorporates broadening followed by deterioration is never truly veins absolutely and to get authentic size of channel.

\section{References}

[1] Sinthanayothin Yuji Hatanaka, Toshiaki Nakagawa, Yoshinori Hayashi, Takeshi Hara and Hiroshi Fujita, "Improvement of Automated Detection Method of Hemorrhages in Fundus Images", 30th Annual International IEEE EMBS Conference Vancouver, British Columbia, Canada, August 20-24, 2008.

[2] Saiprasad Ravishankar, Arpit Jain, Anurag Mittal, "Automated Feature Extraction for Early Detection of Diabetic Retinopathy in Fundus Images", Computer Vision and Pattern Recognition, 2009. CVPR 2009. IEEE Conference on 20-25 June 2009

[3] Garcia, Maria ; Sanchez, Clara I. ; Lopez, Maria I. ; Diez, Ana ; Hornero, Roberto, "Automatic Detection of Red Lesions in Retinal Images Using a Multilayer Perceptron Neural Network", Engineering in Medicine and Biology Society, 2008. EMBS 2008. 30th Annual International Conference of the IEEE 20-25 Aug. 2008.

[4] Langroudi, M.N., Sadjedi, H., "A New Method for Automatic Detection and Diagnosis of Retinopathy Diseases in Colour Fundus Images Based on Morphology", Bioinformatics and Biomedical Technology (ICBBT), 2010 International Conference on 16-18 April 2010

[5] Axel Pinz, Renate Bart1, "Information Fusion in Image Understanding," Pattern Recognition, 1992. Vol.I. Conference A: Computer Vision and Applications, Proceedings., 11th IAPR International Conference on 30 Aug-3 Sep 1992.

[6] María González-Audícana, Xavier Otazu, Octavi Fors, and Jesús Alvarez-Mozos, "A Low Computational-Cost Method to Fuse IKONOS Images Using the Spectral Response Function of Its Sensors," IEEE transactions on geoscience and remote sensing, vol. 44, no. 6, june 2006.

[7] C. Thomas, T. Ranchin, L. Wald, and J. Chanussot, "Synthesis of multispectral images to high spatial resolution: A critical review of fusion methods based on remote sensing physics," IEEE Trans. Geosci. Remote Sens., vol. 46, no. 5, pp. 1301-1312, May 2008.

[8] Duoduo Gou, Tong Ma and Ying Wei, "A novel retinal vessel extraction method based on dynamic scales allocation," 2017 2nd International Conference on Image, Vision and Computing (ICIVC), Chengdu, 2017, pp. 145149, doi: 10.1109/ICIVC.2017.7984535.

[9] Y. Hatanaka et al., "Retinal biometrics based on Iterative Closest Point algorithm," 2017 39th Annual International Conference of the IEEE Engineering in Medicine and Biology Society (EMBC), Seogwipo, 2017, pp. 373-376, doi: 10.1109/EMBC.2017.8036840.

[10] Q. Li et al., "A high-speed end-to-end approach for retinal arteriovenous segmentation," 2017 10th International Congress on Image and Signal Processing, BioMedical Engineering and Informatics (CISP-BMEI), Shanghai, 2017, pp. 1-5, doi: 10.1109/CISP-BMEI.2017.8301975. 